\title{
DISEÑO DE UN MODELO DE ASISTENCIA PERSONAL BREVE PARA PERSONAS CON DISCAPACIDAD INTELECTUAL EN CHILE
}

\section{Design of a short personal assistance model for people with intellectual disabilities in Chile}

\author{
Alberto Minoletti \\ Universidad de Chile. Facultad de Medicina \\ aminoletti2@gmail.com \\ Pamela Gutiérrez \\ Universidad de Chile. Facultad de Medicina \\ M. José Poblete \\ Universidad de Chile. Facultad de Medicina \\ Bernardita López \\ Avanza Inclusión Sociolaboral \\ Juan Bustos \\ Universidad de Chile. Facultad de Medicina \\ Carla MuÑoz \\ Universidad de Chile. Facultad de Medicina \\ Esteban ENCINA \\ Universidad de Chile. Facultad de Medicina
}

Recepción: 4 de febrero de 2020

Aceptación definitiva: 6 de febrero de 2020

Resumen: La asistencia personal para personas con discapacidad intelectual puede contribuir a la aplicación de los nuevos paradigmas de autodeterminación e inclusión 
social y a una mejora de la calidad de vida. Mediante revisión de la literatura y un análisis con expertos profesionales, expertos por experiencia y potenciales usuarios, se elaboró un modelo breve de asistencia personal para ser ejecutado por agentes comunitarios con supervisión profesional. El modelo considera actividades en los espacios habituales de las personas y sus familias, con apoyo para el uso de recursos formales e informales de la comunidad y el refuerzo de redes de apoyo que continúen operando una vez que termine la intervención. Con una duración de 9 meses, el modelo se desarrolla mediante un proceso de 3 etapas: 1) establecimiento de vínculo y elaboración de un plan individualizado, 2) implementación del plan y 3 ) refuerzo de los aprendizajes de los participantes y traspaso a redes de apoyo. Se discute la factibilidad de la aplicación del modelo en países latinoamericanos y la necesidad de realizar estudios que evalúen su implementación.

Palabras clave: discapacidad intelectual; familias; asistente personal; autonomía; vida independiente.

Aвstract: Personal assistance for people with intellectual disabilities can contribute to the application of the new paradigms of self-determination and social inclusion and to an improvement in the quality of life. Through a literature review and analysis with professional experts, experts from experience and potential users, a brief model of personal assistance was developed to be executed by community agents with professional supervision. The model considers activities in the usual spaces of people and their families, with support for the use of community formal and informal resources and the reinforcement of social support networks that continue operating once the intervention ends. With duration of 9 months, the model is developed through a 3 -stage process: 1 ) engagement and formulation of an individualized plan, 2) implementation of the plan, and 3) participants' learning reinforcement and transfer to support networks. The feasibility of the model in Latin American countries and the need for studies to evaluate its implementation are discussed.

KEY WORDs: intellectual disabilities; families; personal assistant; autonomy; independent living.

\section{Introducción}

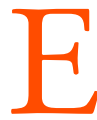

L DESARRollo de Diversas noRmativas y leyes EN Chile ha permitido un progreso leve en el ejercicio de derechos de las personas con discapacidad en los últimos años (Figueroa y Inostroza, 2016; Ministerio de Planificación Chile, 2010). Sin embargo, el abordaje predominante en discapacidad intelectual continúa basado en el modelo biomédico, en el que prima la comprensión de que las causas y consecuencias de la discapacidad se centran en la persona (Palacios y Romañach, 2008). Los estudios realizados en nuestro país muestran que las personas con discapacidad intelectual tienen escasas oportunidades de desarrollar autodeterminación en la toma de decisiones, la formulación de metas y la resolución de problemas; sus sueños y deseos se ven limitados o restringidos "por barreras contextuales, como son la familia, la sobreprotección y la falta de oportunidades” (Vega, Álvarez y Jenaro Río, 2018: 100). 
Los países latinoamericanos requieren avanzar en el campo de la discapacidad intelectual (Lazcano Ponce et al., 2013), implementando los nuevos paradigmas que plantea la Convención de Derechos de las Personas con Discapacidad de la Organización de las Naciones Unidas (2006). Esto implica poner en práctica valores y principios tales como dignidad, igualdad, libertad, inclusión, autodeterminación y calidad de vida de las personas con discapacidad intelectual (Verdugo Alonso, 2011). La Convención ha reconocido en su artículo 19 el derecho a vivir de forma independiente, en su propia comunidad y en igualdad de condiciones con el resto de la población; planteando que esto requiere el acceso a una variedad de servicios de apoyo y a la asistencia personal como una de las herramientas claves (Organización de las Naciones Unidas, 2006). La Observación General núm. 5 del artículo 19 de esta Convención define la asistencia personal como el "apoyo humano dirigido por el interesado o el 'usuario' que se pone a disposición de una persona con discapacidad como un instrumento para permitir la vida independiente"; también, especifica algunos elementos que la diferencian de otros tipos de ayuda personal, tales como su entrega con criterios personalizados, controlada por la persona con discapacidad y con la autogestión de la prestación de los servicios (Comité sobre los Derechos de las Personas con Discapacidad de Naciones Unidas, 2017: 5). Esta Observación General provee un marco conceptual apropiado para el desarrollo de iniciativas de asistencia personal.

Los movimientos de personas con discapacidad en Norteamérica y Europa han contribuido a la toma de conciencia de sus derechos como ciudadanos, promoviendo la asistencia personal, a través de diferentes iniciativas y normativas nacionales, como un modelo que contribuye al logro de valores y principios como los planteados por Verdugo Alonso y apoya las decisiones sobre su existencia y la participación activa en la comunidad (López-Pérez y Ruiz-Seisdedos, 2018). Un ejemplo de esto es lo que realiza la organización Plena inclusión, la que representa en España a las personas con discapacidad intelectual o del desarrollo y contribuye a difundir la asistencia personal, coordinando una red de expertos en el tema, formando a asistentes personales y ayudando a poner en marcha servicios de asistencia personal (Plena inclusión, 2018).

Las diferentes definiciones de asistente personal consideran que este desempeña un servicio que posibilita a las personas con discapacidad llevar una vida independiente, al mismo tiempo que fomenta su autodeterminación y toma de decisiones (Iáñez Domínguez, Aranda Chaves y García Romero, 2018; López-Pérez y Ruiz-Seisdedos, 2018; Plena inclusión, 2018; Suñe y Martínez-García, 2015). El asistente personal proporciona el apoyo necesario para llevar a cabo el proyecto de vida, aumentar el nivel de satisfacción cotidiano, reducir las tensiones que se producen entre la persona con discapacidad y su entorno familiar y facilitar la participación activa y la toma de responsabilidades en la relación de apoyo (Suñe y Martínez-García, 2015). Los resultados obtenidos con la asistencia personal producen un retorno social positivo, que ha sido estimado en España en alrededor de $3 €$ de retorno por cada $1 €$ invertido (Gómez, Moya y Sánchez-Santos, 2015; Iáñez Domínguez et al., 2018).

En discapacidad intelectual, la asistencia personal ha sido utilizada con menor frecuencia que en otras formas de discapacidad y generalmente en casos severos y/o con comorbilidades y discapacidades físicas, mentales o neurológicas (Askheim, 2003). 
A. MINOLETTI, P. GUTIÉRREZ, M. ${ }^{a}$ J. POBLETE, B. LÓPEZ, J. BUSTOS, C. MUÑOZ Y E. ENCINA

Sin embargo, considerando los escasos niveles de autodeterminación, inclusión social y calidad de vida que alcanzan las personas con discapacidad intelectual en Latinoamérica (Lazcano Ponce et al., 2013), la asistencia personal es una prioridad en la región, especialmente en momentos críticos de su desarrollo, como es el período de transición a la vida adulta. El disponer de asistencia personal en estos períodos permitiría a las personas con esta discapacidad mejorar sus trayectorias vitales y lograr una mayor satisfacción con sus vidas.

Las políticas públicas de discapacidad en Chile han iniciado un programa en pequeña escala de tránsito a la vida independiente que incluye la figura del asistente personal para personas con distintos tipos de discapacidades (Rojas Merello, Verdugo Labbe y Troncoso Melo, 2017). Teniendo presente esta ventana de oportunidad y los antecedentes descritos, el presente artículo describe el proceso de diseño de un modelo de asistencia personal para personas con discapacidad intelectual, que pueda ser aplicado en períodos críticos del desarrollo y por un período de tiempo limitado, respondiendo a las necesidades actuales en Chile y en otros países latinoamericanos. La idea es que este modelo pueda ser sometido a estudios cualitativos y cuantitativos y perfeccionado según los resultados, de modo de contribuir eventualmente a las políticas públicas.

\section{Método}

Como una primera etapa para el desarrollo de un modelo de asistencia personal en nuestro país, se decidió focalizar en personas con discapacidad intelectual leve a moderada, especialmente para generar un aprendizaje de cómo superar las barreras de la familia y sociedad en el proceso de avanzar hacia una vida independiente. En una segunda etapa, el modelo se ampliará a todo el universo de personas con discapacidad. El diseño del modelo de asistencia personal consistió en 4 etapas secuenciales: 1) revisión de literatura; 2) elaboración de una primera versión del modelo; 3 ) análisis de la primera versión con expertos profesionales, expertos por experiencia y potenciales usuarios; y 4) elaboración de la versión final.

Etapa 1, revisión de la literatura: Se aplicó el método de "scoping review" (Colquhoun et al., 2014) buscando experiencias y evaluaciones de asistencia personal y programas de apoyo para la vida en la comunidad de personas con discapacidad intelectual leve y moderada. Se revisaron bases de datos PubMed, LILACS e ISI-Web of Science, años 2005 a 2018, y se seleccionaron los artículos en inglés y español que tuvieran conceptos, resultados y conclusiones que pudieran ser aplicables a la asistencia personal de la población diana. Para la sistematización de los resultados se utilizaron 6 dominios (ver apéndice con las referencias para cada dominio), los que se determinaron en base a: a) los principales efectos de los programas de asistencia personal o apoyo encontrados en la literatura, lo cual definió tres dominios (autodeterminación, calidad de vida e inclusión social); b) se consideraron dos dominios de inclusión social, relaciones interpersonales y participación comunitaria, basados en los conceptos 
propuestos por Simplican, Leader, Kosciulek y Leahy (2015); c) la influencia de los padres y cuidadores de personas con discapacidad intelectual, descritos en la literatura como facilitadores y/o obstaculizadores de estos procesos, constituyó el quinto dominio; d) atención de salud, aunque solo dos artículos se referían al tema, se consideró relevante como dominio por el mayor riesgo de morbilidad y mortalidad que presentan las personas con discapacidad intelectual; e) programa de apoyo comunitario de tiempo breve y/o limitado fue el último dominio, basado en la experiencia existente en Chile con intervenciones de salud mental.

Etapa 2, elaboración de una primera versión del modelo: El equipo de investigación seleccionó los aspectos de los siete dominios que parecieron relevantes y factibles de aplicar en nuestra realidad. Uno de los autores, con experiencia en el diseño de modelos de intervención comunitaria, preparó un borrador de modelo de asistencia personal utilizando el marco de la Convención de Derechos de las Personas con Discapacidad y los aspectos seleccionados de los seis primeros dominios del apéndice para definir la forma y contenidos de la intervención; el séptimo dominio contribuyó con el formato de intervención de tiempo limitado y la forma de incorporación de agentes comunitarios como asistentes personales. El borrador fue analizado por el resto del equipo en forma individual y grupal, realizando diversos aportes para mejorar el documento. Las decisiones sobre los principios, objetivos y el método de aplicación propuestos para la primera versión del modelo fueron tomadas en reuniones grupales, buscando consensos entre los integrantes del equipo.

Etapa 3, análisis de la primera versión con expertos: Se realizaron reuniones con 3 grupos independientes entre sí:

- Panel de expertos profesionales: Se consideró como expertos profesionales a personas con título universitario, formación de postgrado en discapacidad intelectual y experiencia reconocida de varios años de trabajo con personas con discapacidad intelectual. Participaron siete expertas que cubrían diferentes áreas de trabajo con personas con discapacidad intelectual, tales como educación, psicología, salud, terapia ocupacional, trabajo social, inclusión laboral y políticas públicas. Sus edades fluctuaban entre 30 y 68 años y su trabajo con personas con discapacidad intelectual se ha desarrollado durante 5 a 35 años. Previo a la reunión, las expertas pudieron revisar el texto de la primera versión del modelo en forma individual, y durante la reunión se revisaron sus distintas secciones, registrándose sus observaciones y sugerencias.

- Entrevista grupal focal con expertos por experiencia: Se consideró como expertos por experiencia a personas con discapacidad intelectual que hubieran participado de organizaciones sociales para la defensa de derechos consagrados en la Convención. El método de entrevista grupal focal se aplicó de acuerdo a la definición de Hernández-Sampieri, Fernández-Collao y Baptista (2014). Asistieron 4 mujeres y 2 hombres, de alrededor de 30 años, que participan en la agrupación de Líderes con 1000 Capacidades y/o en la institución Avanza Inclusión. Posterior al consentimiento informado, se les explicó brevemente 
el modelo de asistencia personal y se les formularon preguntas sobre distintos aspectos de este, utilizando un lenguaje fácil con el apoyo de materiales visuales.

- Entrevista grupal focal con potenciales usuarios de asistencia personal, estudiantes de 19 a 22 años con discapacidad intelectual de un colegio de educación diferencial: Asistieron 2 mujeres y 4 hombres, previo consentimiento informado y asentimiento de sus padres o cuidadores. La metodología fue similar a la utilizada con el grupo anterior.

Etapa 4, elaboración de la versión final del modelo de asistencia personal: Se incorporaron modificaciones basadas en las observaciones y sugerencias de los 3 grupos, con una metodología para el logro de consensos similar a la etapa 2.

\section{Resultados}

\subsection{Resultados de la revisión de literatura}

El único modelo de apoyo para personas con discapacidad intelectual encontrado en la revisión de literatura que tiene definiciones conceptuales y metodológicas y sustento en estudios de efectividad es la "Planificación Centrada en la Persona" (PCP). Si bien no existe en la literatura un estudio controlado aleatorizado, una revisión sistemática de la literatura encontró 11 estudios prospectivos, seis de los cuales incluyeron un grupo de comparación, que muestran que la PCP tiene una serie de impactos positivos de moderada intensidad (Ratti et al., 2016). Una revisión posterior concluye que la PCP tiene beneficios en la vida de las personas con discapacidad intelectual en diferentes áreas, especialmente en la participación en la comunidad, el contacto con los amigos y con la familia y la posibilidad de elecciones en su vida (Rodríguez, García y Fernández, 2018).

Los proponentes de la PCP han formulado definiciones operacionales para los apoyos y las necesidades de apoyo, así como un proceso de intervención con cinco componentes secuenciales: a) identificar lo que la persona más quiere y necesita hacer; b) determinar la forma de apoyo que la persona requiere para lograr lo que ella más quiere y necesita hacer; c) formular un plan de acción para obtener y entregar apoyo; d) iniciar y monitorear el plan, y e) evaluar los resultados personales (Thompson et al., 2009).

La mayoría de los artículos revisados se refieren a estudios transversales -cuantitativos, cualitativos y mixtos- que encuentran asociaciones entre programas de apoyo e indicadores positivos de autonomía o autodeterminación (Björnsdóttir, Stefánsdóttir y Stefánsdóttir, 2015; Frielink, Schuengel y Embregts, 2018; Mauleon, 2018), calidad de vida o bienestar emocional/satisfacción con la vida (Lachapelle et al., 2005) e inclusión social -tanto en sus aspectos de relaciones interpersonales (Pallisera Díaz et al., 2018; Overmars-Marx, Thomése, Verdonschot y Meininger, 2014) como en participación comunitaria (Arvidsson, Granlund, Thyberg y Thyberg, 2014; Kim, Schilling, Kim y Han, 2016; Wilton, Schormans y Marquis, 2018). 
El marco conceptual de la calidad de vida es frecuentemente mencionado en la literatura de discapacidad intelectual como una guía para orientar los servicios de apoyo y para evaluar resultados a través de ocho dimensiones: bienestar emocional, relaciones interpersonales, bienestar material, desarrollo personal, bienestar físico, autodeterminación, inclusión social y derechos (Verdugo Alonso, 2013; Tamarit, 2015).

Como un aspecto complementario, un artículo mostró que las personas con discapacidad intelectual pueden aprender destrezas complejas relacionadas con la autodefensa de sus derechos en salud y con la comunicación, si reciben una capacitación apropiada (Feldman et al., 2012).

Otra línea de trabajo en discapacidad intelectual se refiere a las intervenciones con los padres y cuidadores. La literatura describe experiencias positivas para el desarrollo de actitudes facilitadoras de autonomía en sus hijos/as, así como de beneficios para su propio bienestar emocional, tanto con intervenciones a cargo de profesionales como de actividades de apoyo mutuo entre padres (Heller y Schindler, 2009; Carter et al., 2013; Martínez-Tur, Moliner, Peñarroja, Gracia y Peiró, 2015; Leonard et al., 2016).

En relación a programas de apoyo breves con personas con discapacidad intelectual, se encontraron artículos que describen intervenciones en aspectos limitados de la vida, dos de ellos sobre actividades deportivas (Pérez-Cruzado y Cuesta-Vargas, 2016; Sánchez-Romero, Conesa y Palacios, 2017), uno sobre redes de apoyo para empleo, con base en apoyos comunitarios (familia, voluntarios, organizaciones, etc.), profesionales y entre iguales (Conte y Delgado-Pastor, 2016), y otro sobre destrezas de comunicación y autodefensa en la atención de salud (Feldman et al., 2012). La Intervención en Tiempo Crítico (CTI, Critical Time Intervention), desarrollada en Estados Unidos y aplicada en algunos países latinoamericanos, tiene evidencias de efectividad en la recuperación y reintegración a la comunidad en personas con distintos problemas de salud mental, aunque no se ha probado con personas con discapacidad intelectual (Agrest et al., 2019; Alvarado, Shilling y Jorquera, 2016). CTI incorpora la participación de agentes comunitarios y aborda la integralidad de las necesidades y fortalezas de las personas y sus entornos, estructurando la intervención en un período de 9 meses, con tareas operacionales definidas a lo largo de 3 etapas: a) establecimiento de vínculo y planificación, b) implementación del plan y c) refuerzo y traspaso.

El detalle sobre los resultados relevantes para la asistencia personal encontrados en la revisión de la literatura se muestra en el apéndice al final de este artículo.

\subsection{Resultados del análisis con expertos profesionales, potenciales usuarios y por expertos por experiencia}

Las observaciones del panel de expertos sobre la primera versión del modelo de asistencia personal breve se resumen en tres aspectos principales (otros detalles se muestran en la Tabla 1). En primer lugar, la importancia del abordaje individual con la persona con discapacidad intelectual, que permita conocer sus necesidades y anhelos y apoyarla en la superación de inseguridades para participar en los distintos contextos que desee. Esto implica, según necesidad, la entrega de apoyo tanto para 
A. MiNOLETTI, P. GUTIÉRREZ, M. ${ }^{a}$ J. POBLETE, B. LÓPEZ, J. BUSTOS, C. MUÑOZ Y E. ENCINA

mejorar el desempeño en actividades cotidianas (higiene personal, vestuario, alimentación, transporte, manejo de dinero, manejo de tecnologías de comunicación, etc.) como para la toma de decisiones y la participación en la comunidad en actividades de mayor complejidad.

\begin{tabular}{|c|c|}
\hline \multicolumn{2}{|c|}{$\begin{array}{l}\text { TABLA 1. Principales conclusiones del panel de expertos profesionales sobre } \\
\text { asistencia personal para personas con discapacidad intelectual (DI) } \\
\text { leve o moderada }\end{array}$} \\
\hline Tema & Contenidos \\
\hline $\begin{array}{l}\text { "Escuchar } \\
\text { la voz de la } \\
\text { persona con } \\
\text { DI" }\end{array}$ & $\begin{array}{l}\text { El asistente personal requiere, en primer lugar, comprender las necesidades } \\
\text { y anhelos de la persona que apoya y, posteriormente, puede negociar con la } \\
\text { familia los pasos graduales que se implementarán. Se debe tener claro que } \\
\text { la persona con DI y su familia presentan intereses diferentes. }\end{array}$ \\
\hline $\begin{array}{l}\text { Trabajo con la } \\
\text { familia }\end{array}$ & $\begin{array}{l}\text { Los padres tienden a sobreproteger a las personas con DI durante toda la } \\
\text { vida y a tener una visión negativa sobre sus capacidades. Es importante te- } \\
\text { ner a la familia a favor del trabajo del asistente personal, para lo cual se } \\
\text { requiere graduar la magnitud y velocidad de los cambios. Los avances en } \\
\text { habilidades y autorregulación de la persona con DI pueden contribuir a } \\
\text { disminuir las ansiedades de los padres frente a los cambios. }\end{array}$ \\
\hline $\begin{array}{l}\text { Formación de } \\
\text { los asistentes } \\
\text { personales }\end{array}$ & $\begin{array}{l}\text { La formación debería incluir el conocimiento de los derechos de las perso- } \\
\text { nas con DI y los métodos de accesibilidad cognitiva. Es fundamental que } \\
\text { desarrollen una actitud empática, respetuosa y de valoración de las personas } \\
\text { con DI, sin repetir las actitudes infantilizadoras de la sociedad. }\end{array}$ \\
\hline Apoyo mutuo & $\begin{array}{l}\text { El apoyo mutuo entre familiares de personas con DI contribuye a disminuir } \\
\text { las ansiedades asociadas a los procesos de desarrollo de autonomía en sus } \\
\text { parientes con DI. Similarmente, el apoyo mutuo entre personas con DI fa- } \\
\text { cilita sus procesos de independencia de sus familias. Es importante que los } \\
\text { asistentes personales promuevan actividades grupales orientadas al apoyo } \\
\text { mutuo con ambas poblaciones. }\end{array}$ \\
\hline $\begin{array}{l}\text { El trabajo } \\
\text { del asistente } \\
\text { personal }\end{array}$ & $\begin{array}{l}\text { La asistencia debería promover no solo una vida con mayor independencia } \\
\text { para las personas con DI sino también el desarrollo de la capacidad para } \\
\text { tomar decisiones por sí mismos. El asistente personal tiene que entregar } \\
\text { solo los apoyos estrictamente necesarios, según la voluntad de cada perso- } \\
\text { na. El proceso debería respetar los "tiempos" y los "caminos" individuales. } \\
\text { La asistencia personal tiene que incluir actividades con los entornos de las } \\
\text { personas, contribuyendo a disminuir los obstaculizadores de la escuela, el } \\
\text { trabajo y el barrio. Se sugiere, además, considerar la utilización de aplica- } \\
\text { ciones para teléfonos móviles, las cuales representan un apoyo para diversas } \\
\text { actividades de las personas con DI. }\end{array}$ \\
\hline $\begin{array}{l}\text { Participación } \\
\text { de personas con } \\
\text { DI }\end{array}$ & $\begin{array}{l}\text { Se considera imprescindible su participación en el diseño e implementación } \\
\text { del modelo de asistencia personal. Esto requiere disponer de apoyos para } \\
\text { la participación, primero capacitándolos en algunos conceptos básicos de la } \\
\text { DI y la asistencia personal, y luego facilitando su toma de decisiones en } \\
\text { la construcción de los distintos aspectos del modelo. }\end{array}$ \\
\hline
\end{tabular}


En segundo lugar, se enfatizó la necesidad de trabajar con las familias de las personas con discapacidad intelectual, las cuales generalmente mantienen paradigmas antiguos de exclusión y sobreprotección. Se considera fundamental el acompañamiento a las familias para que superen gradualmente sus miedos, otorguen oportunidades de participación a sus hijos y apoyen procesos de autodeterminación creciente. Las experiencias de participación en grupos de ayuda mutua fueron consideradas cruciales para que las familias asuman los nuevos paradigmas.

Finalmente, se destaca el apoyo que puede entregar la asistencia personal breve en el inicio de la construcción de un proyecto de vida propio, en el cual se debe respetar a las personas con discapacidad intelectual como sujetos de derechos, con una actitud de valoración y respeto.

El grupo de expertos por experiencia que participan en "Líderes con 1000 Capacidades" y/o en "Avanza Inclusión" verbalizaron una variedad de necesidades a las que podría responder el modelo de asistencia personal propuesto por el equipo investigador (ver Tabla 2). Entre ellas destacan el rol que podría jugar el asistente personal para que ellos/ellas alcancen niveles mayores de independencia de sus padres y en el cómo negociar acuerdos apropiados para su edad cronológica. Esta necesidad es mayor en las mujeres, pues consideran que los padres tienen actitudes "machistas" hacia ellas y ejercen un mayor grado de sobreprotección en comparación con los varones. Los temas del manejo de dinero, la continuación de estudios o el acceso a trabajos y la privacidad en relación a su sexualidad fueron también relevantes para el grupo en el sentido de necesitar el apoyo de un asistente personal.

\begin{tabular}{|l|l|}
\hline \multicolumn{2}{|c|}{$\begin{array}{l}\text { TABLA 2. Principales conclusiones de entrevista grupal focal con expertos } \\
\text { por experiencia: personas con discapacidad intelectual (DI) de alrededor } \\
\text { de } 30 \text { años que participan en la organización Líderes con 1000 Capacidades } \\
\text { y/o la institución Avanza Inclusión }\end{array}$} \\
\hline \multicolumn{1}{|c|}{ Dominios } & \multicolumn{1}{c|}{ Necesidades a las que podría responder el asistente personal } \\
\hline $\begin{array}{l}\text { Relación con } \\
\text { los padres }\end{array}$ & $\begin{array}{l}\text { Apoyo en el ejercicio de derechos que conflictúan con los padres, desarro- } \\
\text { llando mayor independencia, aunque manteniendo una actitud respetuosa } \\
\text { hacia ellos. }\end{array}$ \\
\hline $\begin{array}{l}\text { Permisos con } \\
\text { padres }\end{array}$ & $\begin{array}{l}\text { Negociación de acuerdos con padres para tener mayor libertad para salir, } \\
\text { según la edad cronológica, y para la obtención de permisos. También re- } \\
\text { quieren ayuda para que los padres cumplan con este tipo de acuerdos. }\end{array}$ \\
\hline $\begin{array}{l}\text { Actitudes } \\
\text { machistas }\end{array}$ & $\begin{array}{l}\text { Educación de los padres para que modifiquen discriminaciones sobreprotec- } \\
\text { toras con hijas con DI, de modo de igualar el trato entre mujeres y hombres. }\end{array}$ \\
\hline $\begin{array}{l}\text { Manejo de } \\
\text { dinero }\end{array}$ & $\begin{array}{l}\text { Entrenamiento en la organización y administración del dinero y manejo } \\
\text { de situaciones de compras, especialmente con el cálculo del dinero que les } \\
\text { devuelven cuando entregan una cantidad superior al importe. }\end{array}$ \\
\hline $\begin{array}{l}\text { Estudios/ } \\
\text { trabajo }\end{array}$ & $\begin{array}{l}\text { Ayuda para continuar estudios, identificar sus capacidades y vocaciones, y } \\
\text { buscar trabajo remunerado. Además, ayuda para obtener beneficios y pro- } \\
\text { gramas de apoyo para estudio o trabajo. }\end{array}$ \\
\hline Sexualidad & $\begin{array}{l}\text { Apoyo en la defensa de su privacidad y en la modificación de manipulacio- } \\
\text { nes en este tema. }\end{array}$ \\
\hline
\end{tabular}


El grupo de potenciales usuarios que asisten a un colegio de educación diferencial destacaron las principales barreras que encuentran para realizar actividades fuera del hogar y escuela. En general, es en esta última donde se suscitan las mayores instancias de socialización y de exploración de nuevas actividades, y es ahí donde surgen las escasas relaciones de amistad y de pareja. La proyección de estos vínculos a otros contextos se dificulta por las insuficientes habilidades para movilizarse y autocuidarse en la comunidad y por las restricciones que les ponen sus padres. En el caso de las mujeres, todas estas dificultades se acrecientan, manifestando poca confianza en sí mismas y escasa autodeterminación para explorar nuevos ambientes de participación.

Los participantes manifestaron la necesidad de diversificar sus actividades de tiempo libre, más allá de instancias que tengan relación con compras. Al plantearles la figura de la asistencia personal reconocieron la importancia de contar con ella y que les permitiera enriquecer sus vidas y aumentar los espacios de relación con sus pares (ver Tabla 3 para otros detalles de este grupo).

\begin{tabular}{|l|l|}
\hline \multicolumn{2}{|c|}{$\begin{array}{l}\text { TABLA 3. Principales conclusiones de entrevista grupal focal con potenciales } \\
\text { usuarios: estudiantes de } 19 \text { a } 22 \text { años de un colegio de educación diferencial para } \\
\text { personas con discapacidad intelectual (DI) }\end{array}$} \\
\hline \multicolumn{1}{|c|}{ Dominio } & \multicolumn{1}{c|}{ Necesidades a las que podría responder el asistente personal } \\
\hline $\begin{array}{l}\text { Desplazamiento } \\
\text { en la ciudad }\end{array}$ & $\begin{array}{l}\text { Apoyo para la utilización del sistema de transporte público, de modo de } \\
\text { trasladarse a distintos puntos de interés en forma independiente de sus } \\
\text { padres. }\end{array}$ \\
\hline $\begin{array}{l}\text { Actividades de } \\
\text { ocio }\end{array}$ & $\begin{array}{l}\text { Ayuda para participar en una mayor variedad de actividades de entreten- } \\
\text { ción y compras, más allá de las que realizan en la actualidad. Los participan- } \\
\text { tes nombraron una alta gama de actividades posibles, como, por ejemplo, } \\
\text { ir al cine, mall y otros lugares de comercio, lugares donde bailar o comer, } \\
\text { plaza o parque, tomar helados, centro de la ciudad, la vega, zoológico, etc. }\end{array}$ \\
\hline $\begin{array}{l}\text { Riesgos de salir } \\
\text { de la casa }\end{array}$ & $\begin{array}{l}\text { Enseñarles a evitar y manejar los peligros del mundo exterior al hogar. Por } \\
\text { ejemplo, el riesgo de asaltos con pistola u otras armas, robos de celular y } \\
\text { otros objetos, agresiones físicas, etc. }\end{array}$ \\
\hline $\begin{array}{l}\text { Restricciones de } \\
\text { los padres para } \\
\text { salir del hogar }\end{array}$ & $\begin{array}{l}\text { Negociar con los padres permisos más amplios para realizar actividades } \\
\text { fuera del hogar, especialmente para las participantes mujeres. }\end{array}$ \\
\hline $\begin{array}{l}\text { Dependencia de } \\
\text { los padres para } \\
\text { la atención de } \\
\text { salud }\end{array}$ & $\begin{array}{l}\text { Utilización de centros de salud en forma independiente de sus padres u } \\
\text { otros familiares. }\end{array}$ \\
\hline
\end{tabular}

\subsection{Propuesta de Modelo Breve de Asistencia Personal (MOBAP)}

Proponemos una asistencia personal para personas con discapacidad intelectual leve o moderada, en etapa de transición a la vida adulta, basada en PCP y CTI y otros conceptos de la literatura y opinión de expertos. El modelo se orienta a elevar 
autodeterminación, inclusión social y calidad de vida, y sus tres características centrales son: a) se realiza en la comunidad, en los espacios habituales de las personas y sus familias; b) apoya a las personas en el uso de los recursos formales e informales de su comunidad y en el refuerzo de redes de apoyo que continúen operando una vez que termine la intervención; c) promueve la autodeterminación, guiado por los intereses y decisiones de la persona con discapacidad intelectual.

La intervención tiene una duración de 9 meses y la realiza un equipo capacitado para este fin, conformado por asistentes personales y profesionales supervisores:

Asistentes personales: agentes comunitarios que han completado la enseñanza media, tienen conocimientos experienciales de la vida en la comunidad donde viven las personas que apoyan. Su capacitación prioriza la formación ética, el enfoque social de la discapacidad, los derechos de las personas con discapacidad intelectual y su funcionamiento como facilitadores de procesos de cambios en las personas, las familias y las comunidades.

Profesionales supervisores: terapeuta ocupacional, enfermera, trabajador social, y/o psicólogo con formación en programas de salud mental comunitaria. Su función es la supervisión y apoyo al trabajo de los asistentes personales.

El MOBAP incluye los siguientes fundamentos:

- La voz de la persona con discapacidad intelectual es siempre escuchada.

- La persona toma las decisiones del proceso de asistencia personal y el rol del asistente es de apoyo en la toma de decisiones.

- Trabajo en equipo del asistente personal con la persona, compartiendo la responsabilidad de formular, implementar y evaluar el plan de asistencia personal.

- Asistencia individualizada según las características de la persona y su entorno.

- Fortalecimiento de las redes de apoyo social.

- Respeto de los valores, creencias, cultura e identidad de la persona, su familia y su comunidad.

- Asistencia basada en fortalezas, identificando y reforzando las capacidades, conocimientos y recursos de la persona, su familia y su comunidad.

- Apoyo mutuo entre personas con discapacidad intelectual y entre sus familiares.

Se propone que el MOBAP se implemente en tres etapas, de tres meses cada una, de acuerdo a CTI:

Etapa 1: Establecimiento de vínculo y elaboración de un Plan de Asistencia Personal: desarrollo de una relación de confianza y colaboración con la persona con discapacidad intelectual y los familiares principales. Identificación de las experiencias y necesidades importantes para la persona y definición de los apoyos que requiere, con el fin de desarrollar un plan de apoyos individualizados, en base a PCP.

Etapa 2: Implementación del Plan de Asistencia Personal: refuerzo de habilidades y recursos de la persona, familia y comunidad, con el objetivo de ampliar las posibilidades y oportunidades y mejorar la capacidad de respuesta ante potenciales obstáculos y fuentes de estrés. Trabajo de mediación con redes de apoyo para disminuir barreras del entorno y facilitar que continúen operando una vez que el MOBAP termine.

Etapa 3: Refuerzo y transición: refuerzo de los aprendizajes de la persona y de los familiares, promoviendo que continúe su participación social una vez que termine 
la asistencia personal. Al mismo tiempo, existe un refuerzo de la ayuda entre pares, redes de apoyo, recursos comunitarios e instituciones, para los aspectos y momentos que la persona requiera apoyos.

El MOBAP aborda diferentes componentes de la vida diaria de la persona con discapacidad intelectual, según sus intereses, motivaciones, habilidades y decisiones, tales como:

1. Relaciones con la familia: se ayuda a la familia a comprender que la persona, a pesar de algunas limitaciones, tiene pleno derecho a conducir su vida y ejercer el máximo nivel posible de autodeterminación y toma de decisiones. Además, se les apoya a adecuar la comunicación a la velocidad y nivel de complejidad adecuado para la persona.

2. Relaciones con vecinos y amigos: se apoya el inicio y mantenimiento de relaciones sociales con las personas de su interés. De modo que su red de apoyo social opere como fuente de valoración, afectos, apoyo emocional, información, ayuda en la resolución de problemas y ayuda material.

3. Relaciones de pareja: se apoya a la persona a satisfacer sus necesidades de relaciones sentimentales y sexuales con las personas que ella elija. Orientación para una vida sexual saludable (expresión de sentimientos, placer, métodos anticonceptivos, prevención enfermedades de trasmisión sexual, etc.).

4. Actividades recreativas y deportivas: se contribuye a mejorar el conocimiento sobre actividades, programas y organizaciones existentes en la comunidad donde viven. Se refuerzan las habilidades para la utilización de los recursos comunitarios.

5. Actividades artísticas y culturales: se orienta a la persona a identificar y desarrollar sus intereses y talentos y se apoya la participación en actividades y organizaciones dedicadas al arte y cultura.

6. Vida espiritual: se facilita la participación en iglesias e instituciones orientadas a la espiritualidad de su comunidad.

7. Utilización de centros de salud: se facilita la utilización de servicios disponibles, tanto en salud física como mental y el desarrollo de habilidades para comunicarse con profesionales de salud y ejercer sus derechos.

8. Acceso a beneficios sociales que les correspondan: entrega de información y acompañamiento.

9. Trabajo voluntario y remunerado: se apoya en la postulación (ej. acceso a programas de intermediación o apoyo para el trabajo) y desarrollo de habilidades para realizar un trabajo y el mantenimiento de este.

El plan de asistencia debe incluir un máximo de 5 áreas de trabajo y no menos de 3. La idea es cubrir distintos aspectos de la vida, pero por la naturaleza breve de la asistencia se deben priorizar las áreas más relevantes para cada persona.

\section{Discusión}

El modelo de asistencia personal propuesto tiene la fortaleza de basarse tanto en las evidencias y experiencias descritas en la literatura reciente como en la opinión 


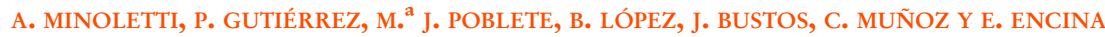

de expertos profesionales que trabajan en el tema y expertos por experiencia que experimentan el significado tener discapacidad intelectual en Chile. Al mismo tiempo, tiene la ventaja de recoger la tradición cultural latinoamericana de apoyos informales de familias, agentes comunitarios, ayuda mutua y agrupaciones locales; lo cual, sumado a la estructura de tiempo limitado, permite reducir el costo de las intervenciones a un valor sustentable para programas sociales orientados a sectores de menores ingresos.

Una de las principales debilidades de esta propuesta es la insuficiente cantidad de estudios nacionales y latinoamericanos que caractericen en mayor profundidad la realidad y percepciones de las personas con discapacidad intelectual leve o moderada en transición a la vida adulta. Menos aún existen estudios que demuestren que los programas de apoyo utilizados en países de mayor desarrollo y realidad sociocultural diferente tienen similares resultados en nuestros contextos. Otra de las debilidades es que se focaliza en las personas con discapacidad leve o moderada.

En concordancia con estas debilidades, el paso siguiente de este proyecto es un estudio de aplicación del MOBAP en una muestra pequeña de personas, para estudiar factibilidad, aceptabilidad, posibles efectos favorables y desfavorables y las percepciones del proceso en los diferentes involucrados. Esto permitirá perfeccionar el modelo y diseñar posteriormente un estudio de efectividad con una muestra adecuada para el tamaño del efecto esperado. En una segunda etapa, se extenderá el modelo a personas con discapacidad intelectual severa.

\section{Referencias bibliográficas}

Agrest, M., Le, P. D., Yang, L. H., Mascayano, F., Alves-Nishioka, S., Dev, S., ... Susser, E. S. (2019). Implementing a community-based task-shifting psychosocial intervention for individuals with psychosis in Chile: perspectives from users. International Journal of Social Psychiatry, 65(1), 38-45. https://doi.org/10.1177/0020764018815204.

Alvarado, R., Shilling, S. y Jorquera, M. J. (2016). Uso de la Intervención Critical Time Intervention en personas con primer episodio de psicosis. Revista de la Facultad de Ciencias Médicas, 72(4), 331-338. https://doi.org/10.31053/1853.0605.v72.n4.13845.

Arvidsson, P., Granlund, M., Thyberg, I. y Thyberg, M. (2014). Important aspects of participation and participation restrictions in people with a mild intellectual disability. Disability and Rehabilitation, 36(15), 1264-1272. https://doi.org/10.3109/09638288.2013.845252.

Askheim, O. P. (2003). Personal assistance for people with intellectual impairments: experiences and dilemmas. Disability and Society, 18(3), 325-339. https://doi.org/10.1080/0968759 032000052897.

BjörnsdótTir, K., STefánsdótTir, G. V. y StefánsdótTir, Á. (2015). «It’s my life»: Autonomy and people with intellectual disabilities. Journal of Intellectual Disabilities, 19(1), 5-21. https://doi.org/10.1177/1744629514564691.

Bowers, K., Corby, D., Lambert, V., Staines, A., McVeigh, T., McKeon, M., ... Sweeney, M. R. (2016). People with intellectual disability and their families' perspectives of Special Olympics Ireland: qualitative findings from the SOPHIE study. Journal of Intellectual Disabilities, 20(4), 354-370. https://doi.org/10.1177/1744629515617059. 


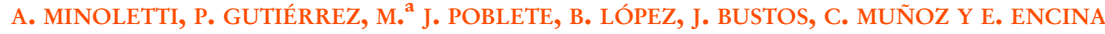

Carter, E., Lane, K., Cooney, M., Weir, K., Moss, C. y Machalicek, W. (2013). Parent assessments of self-determination importance and performance for students with autism or intellectual disability. American Journal on Intellectual and Developmental Disabilities, 118(1), 16-31. https://doi.org/10.1352/1944-7558-118.1.16.

Colquhoun, H. L., Levac, D., O’Brien, K. K., Straus, S., Tricco, A. C., Perrier, L., ... Moher, D. (2014). Scoping reviews: time for clarity in definition, methods, and reporting. Journal of Clinical Epidemiology, 67(12), 1291-1294. https://doi.org/10.1016/j.jclinepi.2014.03.013.

Comité sobre los Derechos de las Personas con Discapacidad de Naciones Unidas (2017). Observación general num. 5 (2017) sobre el derecho a vivir de forma independiente $y$ a ser incluido en la comunidad. Recuperado de http://docstore.ohchr.org/SelfServices/ FilesHandler.ashx?enc $=6 \mathrm{QkG} 1 \mathrm{~d} \% 2 \mathrm{FPPRiCA}$.

Conte, E. V. y Delgado-Pastor, L. C. (2016). Un estudio sobre la eficacia en la estructuración de los apoyos en formación profesional para jóvenes con discapacidad intelectual. Siglo Cero, 47(2), 99-114. https://doi.org/10.14201/scero201647299114.

Crawford, C., Burns, J. y Fernie, B. A. (2015). Psychosocial impact of involvement in the Special Olympics. Research in Developmental Disabilities, 45-46, 93-102. https://doi.org/ 10.1016/j.ridd.2015.07.009.

EtXeverria, X. (2018). Proyectos de vida y acompañamiento a las personas con discapacidad intelectual: enfoque ético. Siglo Cero, 49(4), 35-50. https://doi.org/10.14201/scero20184943550.

Feldman, M. A., Owen, F., Andrews, A., Hamelin, J., Barber, R. y Griffiths, D. (2012). Health self-advocacy training for persons with intellectual disabilities. Journal of Intellectual Disability Research, 56(11), 1110-1121. https://doi.org/10.1111/j.1365-2788.2012.01626.x.

Figueroa, M. e Inostroza, H. (2016). Discapacidad y salud mental: una visión desde SENADIS. Recuperado de https://www.senadis.gob.cl/descarga/i/3179/documento.

Frielink, N., Schuengel, C. y Embregts, P. J. C. M. (2018). Autonomy support, need satisfaction, and motivation for support among adults with intellectual disability: testing a self-determination theory model. American Journal on Intellectual and Developmental Disabilities, 123(1), 33-49. https://doi.org/10.1352/1944-7558-123.1.33.

Gómez, N., Moya, M. J. y Sánchez-Santos, F. (2015). Financiación y rentabilidad socioeconómica de la asistencia personal. En E. Ortega-Alonso (Coord.), La situación de la asistencia personal en España (pp. 145-147). Recuperado de http://www.predif.org/sites/ default/files/documents/ASISTENCIA\%20PERSONAL.pdf.

Heller, T. y Schindler, A. (2009). Family support interventions for families of adults with intellectual and developmental disabilities. International Review of Research in Mental Retardation, 37, 299-332. https://doi.org/10.1016/S0074-7750(09)37009-3.

Hernández-Sampieri, R., Fernández-Collao, C. y Baptista, P. (2014). Metodología de la investigación (6. $\left.{ }^{\mathrm{a}}\right)$. México: Mc-Graw Gill.

Hillman, A., Donelly, M., Whitaker, L., Dew, A., Stancliffe, R. J., Knox, M., ... ParmenTER, T. R. (2012). Experiencing rights within positive, person-centred support networks of people with intellectual disability in Australia: experiencing rights. Journal of Intellectual Disability Research, 56(11), 1065-1075. https://doi.org/10.1111/j.1365-2788.2012.01647.x.

Iáñez Domínguez, A., Aranda Chaves, J. L. y García Romero, J. (2018). Impacto económico y social de la asistencia personal a través de la metodología del Retorno Social de la Inversión. Siglo Cero, 6(2), 81-102. Recuperado de https://www.cedd.net/redis/index.php/ redis/article/view/425.

Iriarte, E., O’Brien, P., McConkey, R., Wolfe, M. y O’Doherty, S. (2014). Identifying the key concerns of Irish persons with intellectual disability. Journal of Applied Research in Intellectual Disabilities, 27, 564-575. https://doi.org/10.1111/jar.12099. 


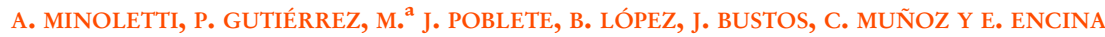

Kim, J., Schilling, M. L., Kim, M. y Han, A. (2016). Contribution of leisure satisfaction, acceptance disability, and social relationship to life satisfaction among Korean individuals with intellectual disability. Journal of Mental Health Research in Intellectual Disabilities, 9(3), 157-170. https://doi.org/10.1080/19315864.2016.1182237.

KripKe, C. (2016). Supported health care decision-making for people with intellectual and cognitive disabilities. Family Practice, 33(5), 445-446. https://doi.org/10.1093/fampra/cmw060.

Lachapelle, Y., Wehmeyer, M. L., Haelewyck, M.-C., Courbois, Y., Keith, K. D., SchaLOCK, R., ... WALSH, P. N. (2005). The relationship between quality of life and self-determination: an international study. Journal of Intellectual Disability Research, 49(10), 740744. https://doi.org/10.1111/j.1365-2788.2005.00743.x.

Lazcano Ponce, E., Katz, G., Allen Leigh, B., Magaña Valladares, L., Rangel Eudave, G., Minoletti Scaramelli, A., ... Salvador Carulla, L. (2013). Trastornos del desarrollo intelectual en América Latina: un marco para establecer las prioridades políticas de investigación y atención. Revista Panamericana de Salud Pública, 34(3), 204-209. Recuperado de https://scielosp.org/pdf/rpsp/2013.v34n3/204-209/es.

Leonard, H., Foley, K.-R., Pikora, T., Bourke, J., Wong, K., McPherson, L., ... Downs, J. (2016). Transition to adulthood for young people with intellectual disability: the experiences of their families. European Child and Adolescent Psychiatry, 25(12), 1369-1381. https://doi.org/10.1007/s00787-016-0853-2.

Lombardi, M., Croce, L., Claes, C., Vandevelde, S. y Schalock, R. L. (2016). Factors predicting quality of life for people with intellectual disability: results from the ANFFAS study in Italy. Journal of Intellectual and Developmental Disability, 41(4), 338-347. https:// doi.org/10.3109/13668250.2016.1223281.

López-PÉrez, M. y Ruiz-Seisdedos, S. (2018). Asistencia personal: equiparación de derechos y oportunidades. Comparativa entre Suecia y España. Siglo Cero, 6(2), 103-130. Recuperado en http://riberdis.cedd.net/handle/11181/5562.

Martínez-Tur, V., Moliner, C., Peñarroja, V., Gracia, E. y Peiró, J. M. (2015). From service quality in organisations to self-determination at home. Journal of Intellectual Disability Research, 59(10), 882-890. https://doi.org/10.1111/jir.12190.

Ministerio de Planificación Chile (2010). Ley 20422: Establece Normas sobre Igualdad de Oportunidades e Inclusión Social de Personas con Discapacidad. Recuperado de http:// www.leychile.cl/N?i=1010903\&f=2010-02-10\&p=.

Neuman, R. y Reiter, S. (2017). Couple relationships as perceived by people with intellectual disability - implications for quality of life and self-concept. International Journal of Developmental Disabilities, 63(3), 138-147. https://doi.org/10.1080/20473869.2016.1173422.

Organización de las Naciones Unidas (2006). Convención sobre los Derechos de las Personas con Discapacidad. https://doi.org/10.18356/92a7700f-es.

Overmars-Marx, T., Thomése, F., Verdonschot, M. y Meininger, H. (2014). Advancing social inclusion in the neighbourhood for people with an intellectual disability: an exploration of the literature. Disability and Society, 29(2), 255-274. https://doi.org/10.1080/096 87599.2013.800469.

Palacios, A. y Romañach, J. (2008). El modelo de la diversidad: una nueva visión de la bioética desde la perspectiva de las personas con diversidad funcional (discapacidad). Intersticios: Revista Sociológica de Pensamiento Crítico, 2(2), 37-47. Recuperado de https://www. intersticios.es/article/view/2712.

Pallisera Díaz, M. P., Noell, J. F., Rovira, C. P., Suñé, M. V., Gabernet, M. J. V., GaroLera, G. D. y Belmonte, M. C. (2018). Retos para la vida independiente de las personas con discapacidad intelectual. Un estudio basado en sus opiniones, las de sus familias y las de 
los profesionales. Siglo Cero, 6(1), 7-29. Recuperado de https://www.cedd.net/redis/index. $\mathrm{php} /$ redis/article/view/370.

Pelletier, J. E. y Joussemet, M. (2017). The benefits of supporting the autonomy of individuals with mild intellectual disabilities: an experimental study. Journal of Applied Research in Intellectual Disabilities, 30(5), 830-846. https://doi.org/10.1111/jar.12274.

Pérez-Cruzado, D. y Cuesta-Vargas, A. I. (2016). Changes on quality of life, self-efficacy and social support for activities and physical fitness in people with intellectual disabilities through multimodal intervention. European Journal of Special Needs Education, 31(4), 553-564. https://doi.org/10.1080/08856257.2016.1187876.

Plena inclusión (2018). Asistencia Personal. Una herramienta clave para el ejercicio del derecho a la Vida Independiente de las personas con discapacidad intelectual o del desarrollo. Madrid: Plena inclusión España. Recuperado de http://www.plenainclusion.org/sites/default/files/asistenciapersonal.pdf.

Power, A. (2008). Caring for independent lives: geographies of caring for young adults with intellectual disabilities. Social Science and Medicine, 67(5), 834-843. https://doi.org/10.1016/j. socscimed.2008.05.023.

Ratti, V., Hassiotis, A., Crabtree, J., Deb, S., Gallagher, P. y Unwin, G. (2016). The effectiveness of person-centred planning for people with intellectual disabilities: a systematic review. Research in Developmental Disabilities, 57, 63-84. https://doi.org/10.1016/j. ridd.2016.06.015.

Rodríguez, T. C., García, M. T. I. y Fernández, S. F. (2018). Efectos y beneficios de la Planificación Centrada en la Persona (PCP) en las personas adultas con discapacidad intelectual, en las organizaciones y en la comunidad. Estado de la cuestión. Siglo Cero, 48(2), 25-40-40. https://doi.org/10.14201/scero20174822540.

Rojas Merello, A., Verdugo labbe, C. y Troncoso Melo, S. (2017). Servicios de apoyo para la vida independiente desde una perspectiva de derecho, autonomía y calidad de vida. Una experiencia de SENADIS. Revista Latinoamericana en Discapacidad, Sociedad y Derechos Humanos, 1(2), 44-59. Recuperado de http://redcdpd.net/revista/index.php/revista/ article/view/55/21.

Sánchez-Romero, E. I., Conesa, M. P. V. y Palacios, C. de F. (2017). La promoción de valores a través de un programa de actividad física y predeportes en personas con discapacidad intelectual leve. Siglo Cero, 48(3), 73-86-86. https://doi.org/10.14201/scero20174837386.

Shogren, K. A. (2013). A social-ecological analysis of the self-determination literature. Intellectual and Developmental Disabilities, 51(6), 496-511. https://doi.org/10.1352/19349556-51.6.496.

Simplican, S. C., Leader, G., Kosciulek, J. y Leahy, M. (2015). Defining social inclusion of people with intellectual and developmental disabilities: an ecological model of social networks and community participation. Research in Developmental Disabilities, 38, 18-29. https://doi.org/10.1016/j.ridd.2014.10.008.

Suñe, A. y Martínez-García, I. (2015). La figura del asistente personal. En E. Ortega-Alonso (Coord.), La situación de la asistencia personal en España (pp. 18-23). Recuperado de http:// www.predif.org/sites/default/files/documents/ASISTENCIA\%20PERSONAL.pdf.

TAMARIT, J. (2015). La transformación de los servicios hacia la calidad de vida. Una iniciativa de innovación social de FEAPS. Siglo Cero, 46(3), 47-71. https://doi.org/10.14201/ scero20154634771.

Thompson, J. R., Bradley, V. J., Buntinx, W. H. E., Schalock, R. L., Shogren, K. A., SNell, M. E., ... YEAgER, M. H. (2009). Conceptualizing supports and the support needs 
A. MiNOLETTI, P. GUTIÉRREZ, M. ${ }^{a}$ J. POBLETE, B. LÓPEZ, J. BUSTOS, C. MUÑOZ Y E. ENCINA

of people with intellectual disability. Intellectual and Developmental Disabilities, 47(2), 135-146. https://doi.org/10.1352/1934-9556-47.2.135.

Vega, V., Álvarez, I. y Jenaro, C. (2018). Autodeterminación: explorando las autopercepciones de adultos con síndrome de Down chilenos. Siglo Cero, 49(2), 89-104. https://doi.org/ $10.14201 /$ scero201849289104.

Verdugo, M. Á. (2011). Dignidad, igualdad, libertad, inclusión, autodeterminación y calidad de vida. Siglo Cero, 42(240), 18-23. Recuperado de http://riberdis.cedd.net/handle/11181/3772.

Verdugo, M. Á. (2013). Escala INICO-FEAPS: Evaluación integral de la calidad de vida de personas con discapacidad intelectual o del desarrollo. Salamanca: Publicaciones del INICO.

Wilton, R., Schormans, A. F. y Marquis, N. (2018). Shopping, social inclusion and the urban geographies of people with intellectual disability. Social E Cultural Geography, 19(2), 230-252. https://doi.org/10.1080/14649365.2016.1274773. 


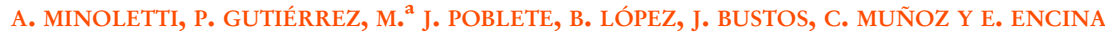

\section{APÉNDICE}

\begin{tabular}{|c|c|}
\hline Dominios & Principales conceptos pertinentes para la asistencia personal \\
\hline 1. Autodeterminación & $\begin{array}{l}\text { Asociación significativa entre actividades de apoyo a la autonomía y } \\
\text { la satisfacción de necesidades y el bienestar subjetivo (Frielink et al., } \\
\text { 2018). } \\
\text { Distintos programas de apoyo y/o intervenciones para facilitar el } \\
\text { desarrollo de autonomía o autodeterminación en personas con DI } \\
\text { han mostrado resultados favorables (Björnsdóttir et al., 2015; Pelle- } \\
\text { tier y Joussemet, 2017; Shogren, 2013). } \\
\text { Aspectos ético del apoyo a las personas con discapacidad intelectual } \\
\text { en el ejercicio de su autonomía (Mauleon, 2018). }\end{array}$ \\
\hline $\begin{array}{l}\text { 2. Calidad de vida } \\
\text { (bienestar emocional } \\
\text { o satisfacción con la } \\
\text { vida) }\end{array}$ & $\begin{array}{l}\text { Importancia del desarrollo de la autodeterminación para mejorar la } \\
\text { calidad de vida (Lachapelle et al., 2005). } \\
\text { Programas de apoyo basados en la Planificación Centrada en la } \\
\text { Persona han mostrado efectos positivos en la calidad de vida de } \\
\text { personas con DI en estudios prospectivos de efectividad (Lombar- } \\
\text { di, Croce, Claes, Vandevelde y Schalock, 2016; Ratti et al., 2016; } \\
\text { Thompson et al., 2009; Rodríguez et al., 2018). } \\
\text { Marco conceptual de la calidad de vida para orientar los servicios de } \\
\text { apoyo y evaluar resultados (Verdugo Alonso, 2013; Tamarit, 2015). }\end{array}$ \\
\hline $\begin{array}{l}\text { 3. Inclusión social: } \\
\text { relaciones } \\
\text { interpersonales }\end{array}$ & $\begin{array}{l}\text { Importancia del apoyo para establecer relaciones personales y socia- } \\
\text { les significativas (Pallisera Díaz et al., 2018). } \\
\text { El vecindario puede actuar tanto como facilitador o barrera para la } \\
\text { inclusión social (Overmars-Marx et al., 2014). } \\
\text { Las personas con DI que participan en una relación de pareja tienen } \\
\text { mayor la calidad de vida (Neuman y Reiter, 2017). }\end{array}$ \\
\hline $\begin{array}{l}\text { 4. Inclusión social: } \\
\text { participación } \\
\text { comunitaria }\end{array}$ & $\begin{array}{l}\text { El nivel de participación en actividades y la importancia que estas } \\
\text { representan para la persona se asocian positivamente con el nivel de } \\
\text { bienestar subjetivo (Arvidsson et al., 2014). } \\
\text { La participación en actividades de tiempo libre (ocio) contribuye a } \\
\text { mejorar el nivel de satisfacción con la vida (Kim et al., 2016). } \\
\text { La participación en actividades deportivas ha mostrado varios efec- } \\
\text { tos positivos en personas con DI (Bowers et al., 2016; Crawford, } \\
\text { Burns y Fernie, 2015; Pérez-Cruzado y Cuesta-Vargas, 2016). } \\
\text { Las visitas con apoyo a centros comerciales contribuyen a satisfacer } \\
\text { necesidades de las personas con DI (Wilton et al., 2018). } \\
\text { Las personas con DI valoran el empleo como una fuente de ocupa- } \\
\text { ción, satisfacción y establecimiento de relaciones sociales y amista- } \\
\text { des (Iriarte, O’Brien, McConkey, Wolfe y O’Doherty, 2014). } \\
\text { Los riesgos en la administración del dinero limitan la independencia } \\
\text { y participación de las personas con DI (Iriarte et al., 2014). }\end{array}$ \\
\hline
\end{tabular}




\begin{tabular}{|l|l|}
\hline \multicolumn{1}{|c|}{ Dominios } & \multicolumn{1}{|c|}{ Principales conceptos pertinentes para la asistencia personal } \\
\hline $\begin{array}{l}\text { 5. Relaciones con } \\
\text { padres y cuidadores }\end{array}$ & $\begin{array}{l}\text { La sobreprotección de padres y cuidadores contribuye a limitar la } \\
\text { autonomía e independencia de las personas con DI (Power, 2008; } \\
\text { Björnsdóttir et al., 2015). } \\
\text { Experiencias positivas con programas de apoyo a padres en el desa- } \\
\text { rrollo de actitudes facilitadoras de autonomía (Leonard et al., 2016; } \\
\text { Carter et al., 2013; Heller y Schindler, 2009; Martínez-Tur et al., } \\
\text { 2015). } \\
\text { Las familias y los cuidadores pueden ser tanto barreras como fa- } \\
\text { cilitadores del ejercicio de derechos humanos (Kripke, 2016; Hill- } \\
\text { man et al., 2012). }\end{array}$ \\
\hline $\begin{array}{l}\text { 6. Salud } \\
\text { Los médicos habitualmente no les hablan a las personas con DI sino } \\
\text { a los familiares o cuidadores que las acompañan (Iriarte et al., 2014). } \\
\text { Existe evidencia de que las personas con DI pueden aprender des- } \\
\text { trezas complejas relacionadas con la autodefensa de la salud y la co- } \\
\text { municación con el personal de salud (Feldman et al., 2012). }\end{array}$ \\
\hline $\begin{array}{l}\text { Apoyo comunitario tiempo breve y/o } \\
\text { limitado }\end{array}$ & $\begin{array}{l}\text { Algunos artículos describen intervenciones breves sobre actividades } \\
\text { deportivas (Pérez-Cruzado y Cuesta-Vargas, 2016; Sánchez-Rome- } \\
\text { ro et al., 2017), redes de apoyo comunitario para empleo (Conte y } \\
\text { Delgado-Pastor, 2016) y destrezas de comunicación y autodefensa } \\
\text { en la atención de salud (Feldman et al., 2012). } \\
\text { La Intervención en Tiempo Crítico (CTI, Critical Tíme Interven- } \\
\text { tion) tiene evidencias de efectividad en la recuperación y reintegra- } \\
\text { ción a la comunidad de personas con distintos problemas de salud } \\
\text { mental, aunque no ha sido aplicada en personas con DI (Agrest } \\
\text { et al., 2019). } \\
\text { La utilización del modelo CTI ejecutado por agentes comunitarios } \\
\text { ha mostrado adecuada factibilidad y aceptabilidad en Chile (Agrest } \\
\text { et al., 2019; Alvarado et al., 2016). }\end{array}$ \\
\hline
\end{tabular}




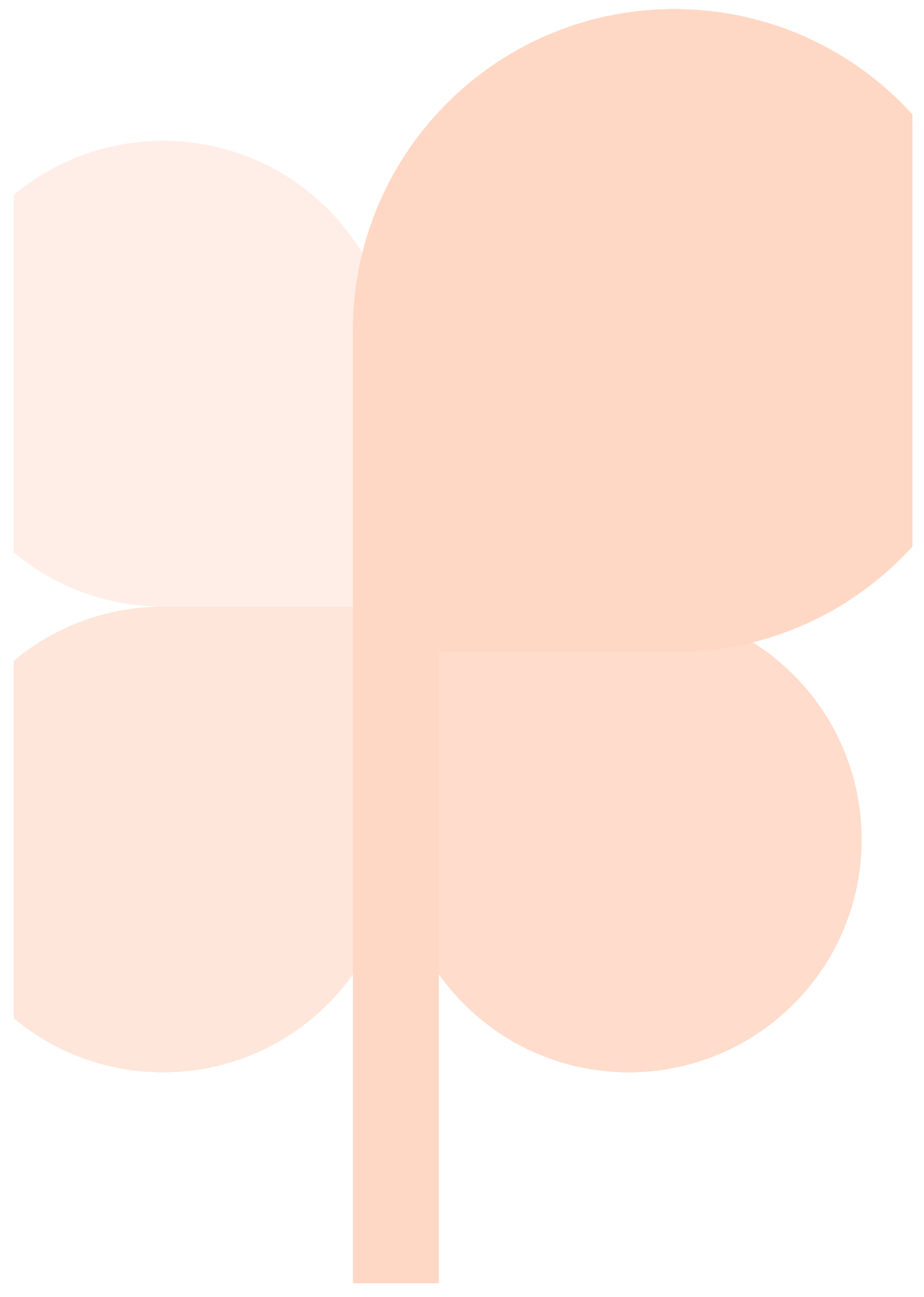

